\title{
Dexamethasone exerts profound immunologic interference on treatment efficacy for recurrent
} glioblastoma

E T Wong, E Lok, S Gautam and K D Swanson

Correction to: Br J Cancer (2015) 113, 232-241; doi:10.1038/bjc.2015.238

Following publication of the above paper in issue 113, number 2 of the journal, the authors spotted a number of errors, two relating to Figure 1, and one relating to Table 1 . There is one further typographical error in the text.

On page 3, Figure 1, section 1D: the heading for the y-axis should add '(' before ' $\mathrm{cm}^{2}$ ', as in 'Tumor size $\left(\mathrm{cm}^{2}\right)$ '.

(C) 2015 Cancer Research UK. All rights reserved 0007 - 0920/15
Again on page 3, Figure 1, legend, lines 12-13: there is a typographical error and '11.2' should be '106.1'. The sentence should read as '...had a median tumour size of 4.2 (range $0.0-106.1$ ) $\mathrm{cm}^{2}$ as compared with ....'

On Table 1, under heading of Absolute T-cell subsets: '+' in superscript should appear after CD3, CD4 and $\mathrm{CD} 8$, as in 'CD3 ${ }^{+}$', ' $\mathrm{CD} 4{ }^{+}$' and 'CD8 ${ }^{+}$'.

On page 7, left column, line 2: 'C3' should be 'CD3'.

\section{BJC}

Granulocyte colony-stimulating factor receptor signalling via Janus kinase 2/signal transducer and activator of transcription 3 in ovarian cancer

J Kumar, F W Fraser, C Riley, N Ahmed, D R McCulloch and A C Ward

Correction to: British Journal of Cancer (2014) 110, 133-145; doi:10.1038/ bjc. 2013.673

The authors have become aware of an error in Figure 1 in which one of the images (panel E, $\alpha$-G-CSFR) was inadvertently duplicated (in panel $\mathrm{H}$, $\alpha$-pSTAT3). The corrected Figure 1 is reproduced below. 